\title{
Nonlinear diffusion process in a Bénard system at the critical point for the onset of convection
}

\author{
R. A. Kraenkel, S. M. Kurcbart, and J. G. Pereira \\ Instituto de Física Teórica, Universidade Estadual Paulista, Rua Pamplona 145, 01405-900 São Paulo, São Paulo, Brazil
}

M. A. Manna

Laboratoire de Physique Mathématique, Universite de Montpellier II, 34095 Montpellier CEDEX 05, France

(Received 22 December 1992)

\begin{abstract}
The evolution equation governing surface perturbations of a shallow fluid heated from below at the critical Rayleigh number for the onset of convective motion, and with boundary conditions leading to zero critical wave number, is obtained. A solution for negative or cooling perturbations is explicitly exhibited, which shows that the system presents sharp propagating fronts.
\end{abstract}

PACS number(s): 47.20.Bp

The onset of convective motion in systems of the Rayleigh-Bénard type has been a subject of investigations for many years. For any given thermal and mechanical boundary conditions one may determine, through a linear stability analysis, the critical Rayleigh number above which the quiescent state is no longer stable [1]. Furthermore, a characteristic wavelength is also obtained, related to the spacing of convective cells. Both the critical number and the wavelength depend strongly on the boundary conditions. A peculiar phenomenon is that, in some cases, the characteristic wavelength may be infinite. In this paper we will study one such system with the fluid bounded below by a rigid plate and above by a free deformable surface, with constant heat flux imposed on both the upper and lower boundaries. However, instead of applying the linear stability analysis, we will resort to techniques inspired in the theory of nonlinear waves. Since we know beforehand that the instability is associated with large space scales, it is quite natural to look for the limit of long-wavelength disturbances in shallow water [2]. In this way, we will be able to obtain not only the critical Rayleigh number for the onset of convection (already known to be $R=320$ from the linear analysis [1]), but also the equation governing the time evolution of both surface displacement and temperature disturbance at the critical point, a problem that cannot be tackled through a linear approach. A similar procedure has already been used [3] to study the same system, but with an undeformable upper surface, in a slightly supercritical regime, that is for $R>320$. Our results, on the other hand, describe the situation just at the critical point $R=320$, but before convection could set in, when diffusion still dominates.

To implement the above-mentioned procedure, we will use the reductive perturbation method of Taniuti $[4,5]$. This method has been extensively applied to the study of long waves in a variety of physical systems [6,7]. More recently, it has been applied to systems of the RayleighBénard [8,9] and Bénard-Marangoni [10] types where, with different boundary conditions from those used in the present work, the Korteweg-de Vries, KadomtsevPetviashvili, and Burgers equations have been obtained as the equations governing surface long-wavelength perturbations. However, it should be kept in mind that the use of multiple scales, as in the reductive perturbation method, does not necessarily lead to equations describing wave disturbances. In fact, as we shall see in what follows, the phenomenon we are going to describe is not related to oscillatory motion. Instead, it is a diffusive phenomenon governed by a nonlinear diffusion equation, as the one discussed by Zel'dovich and Raizer [11] in a different context. For a negative surface, or a cooling temperature perturbation, we predict the existence of sharp propagating fronts. For a positive or heating perturbation, our approach is not able to give an answer to the problem since, in this case, the system enters the convective regime, and the scaling we are going to use is not appropriate to describe this phenomenon.

Let us then consider a fluid which, when at rest, lies between $z=0$ and $z=h$. We idealize the fluid to be twodimensional, and work in the Boussinesq approximation. At the bottom it is limited by an insulating plate, with no-slip boundary conditions. At the upper surface, which is supposed to be free and deformable, a constant heat flux is imposed. The fluid is unbounded in the horizontal direction, and it is acted upon by gravity, with $\mathbf{g}=-g \mathbf{z}$. We will take into account only buoyancy effects, disregarding those coming from surface tension. The velocity will be denoted by $\mathbf{v}=(u, 0, w)$, the pressure by $p$, the temperature by $T$, and the density by $\rho$ which is supposed to depend linearly on the temperature,

$$
\rho=\rho_{0}\left[1-\alpha\left(T-T_{0}\right)\right]
$$

where $\alpha$ is a constant, and $T_{0}$ and $\rho_{0}$ are reference values for the temperature and density, respectively. The equations governing the fluid motion are the following:

$$
\begin{aligned}
& u_{x}+w_{z}=0, \\
& \rho_{0}\left(u_{t}+u u_{x}+w u_{z}\right)=-p_{x}+\mu\left(u_{x x}+u_{z z}\right), \\
& \rho_{0}\left(w_{t}+u w_{x}+w w_{z}\right)=-p_{z}+\mu\left(w_{x x}+w_{z z}\right)-g \rho, \\
& T_{t}+u T_{x}+w T_{z}=\kappa\left(T_{x x}+T_{z z}\right) .
\end{aligned}
$$


In the above expressions, the subscripts denote partial derivatives, $\mu$ is the viscosity, and $\kappa$ is the thermal diffusivity. We now have to state the boundary conditions. On the upper free surface $z=h+\eta(x, t)$, we have [12]

$$
\begin{aligned}
& \eta_{t}+u \eta_{x}=w \\
& p-p_{a}-\frac{2 \mu}{N^{2}}\left[w_{z}+u_{x}\left(\eta_{x}\right)^{2}-\eta_{x}\left(u_{z}+w_{x}\right)\right]=0 \\
& {\left[1-\left(\eta_{x}\right)^{2}\right]\left(u_{z}+w_{x}\right)+2 \eta_{x}\left(w_{z}-u_{x}\right)=0} \\
& -\eta_{x} T_{x}+T_{z}=-\frac{F}{k}
\end{aligned}
$$

where $N=\left[1+\left(\eta_{x}\right)^{2}\right]^{1 / 2}, F$ is the constant heat flux through the upper surface, $k$ is the thermal conductivity, and $p_{a}$ is the constant pressure exerted on the upper free surface. On the lower surface $z=0$, the boundary conditions are the following:

$$
\begin{aligned}
& w=u=0, \\
& T_{z}=-(F / k) .
\end{aligned}
$$

We will consider small disturbances from the static solution, which is given by

$$
\begin{aligned}
& T_{s}=T_{0}-\frac{F}{k}(z-h), \\
& p_{s}=p_{a}-g \rho_{0}\left[(z-h)+\frac{\alpha F}{2 k}(z-h)^{2}\right], \\
& \rho_{s}=\rho_{0}\left[1+\frac{\alpha F}{k}(z-h)\right] .
\end{aligned}
$$

Before proceeding further, let us introduce appropriate units: $h$ for space, $h^{2} / \kappa$ for time, $F h / k$ for temperature, and $\rho_{0} h^{3}$ for mass. All equations can thus be put in a nondimensional form. In this process, the following dimensionless numbers will appear: the Prandtl number $\sigma=\mu / \rho_{0} \kappa$, the Rayleigh number $R=\rho_{0} g \alpha F h^{4} / k \kappa \mu$, and the Galileo number $G=g h^{3} \rho_{0}^{2} / \mu^{2}$. From now on, all quantities will be understood as nondimensional.

We are now in a position to introduce the reductive perturbation method. First we define the stretched coordinates according to

$$
\xi=\epsilon x, \quad \tau=\epsilon^{3} t
$$

with $\epsilon$ a small dimensionless parameter. Usually, the Gardner-Morikawa transformation also involves a group velocity $c$ in the definition of the new variable $\xi$. However, if we introduce it here, it turns out to be zero when the calculations are performed. This is a clear indication that the instability we are considering is nonoscillatory, and it is the reason we have dropped the velocity $c$ from the definition of $\xi$. A word should also be said about the time scaling. Depending on mechanical and thermal boundary conditions, this scaling may give rise to dispersive oscillatory phenomena [8]. Inspired by this fact, but choosing other boundary conditions, we will be able to describe in this paper a different phenomenon, which will not be related to oscillatory motion. However, we should mention that the above stretching is not unique. For example, in Ref. [13] a different one has been used to study the convective regime of a system with the same boundary conditions.

Now, we expand all dependent variables in the following way:

$$
\begin{aligned}
& u=\epsilon^{2}\left(u_{0}+\epsilon u_{1}+\cdots\right), \\
& w=\epsilon^{3}\left(w_{0}+\epsilon w_{1}+\cdots\right), \\
& p-p_{s}=\epsilon\left(p_{0}+\epsilon p_{1}+\cdots\right), \\
& T-T_{s}=\epsilon\left(\Theta_{0}+\epsilon \Theta_{1}+\cdots\right), \\
& \eta=\epsilon\left(\eta_{0}+\epsilon \eta_{1}+\cdots\right) .
\end{aligned}
$$

Once we have substituted these expressions in Eqs. (1)-(11), a well-defined perturbative scheme shows up, and we can solve those equations order by order in $\epsilon$. We will not, however, present the calculation in full detail as they are quite extensive, although straightforward. Let us rather state the results of each order. In the lowest order we have

$$
\begin{aligned}
& \Theta_{0}=\frac{8 G \sigma}{3 R} \eta_{0} \\
& p_{0}=G \sigma^{2}\left(\frac{8 z}{3}-\frac{5}{3}\right) \eta_{0} \\
& u_{0}=G \sigma\left[\frac{4 z^{3}}{9}-\frac{5 z^{2}}{6}+\frac{z}{3}\right) \eta_{0 \xi}, \\
& w_{0}=-G \sigma\left(\frac{z^{4}}{9}-\frac{5 z^{3}}{18}+\frac{z^{2}}{6}\right) \eta_{0 \xi \xi} .
\end{aligned}
$$

At this order, $\eta_{0}(\xi, \tau)$ is an arbitrary function. The next order gives

$$
\begin{aligned}
\Theta_{1}= & g(\xi, \tau), \\
p_{1}= & R \sigma(z-1) g+G \sigma^{2} \eta_{1}+\left(\frac{R \sigma}{2}-\frac{8 G \sigma^{2}}{3}\right] \eta_{0}^{2}, \\
u_{1}= & R\left[\frac{z^{3}}{6}-\frac{z^{2}}{2}+\frac{z}{2}\right) g_{\xi}+G \sigma\left(\frac{z^{2}}{2}-z\right) \eta_{1 \xi} \\
& +\frac{1}{2}\left[\left(R-\frac{16 G \sigma}{3}\right)\left[\frac{z^{2}}{2}-z\right)-G \sigma z\right]\left(\eta_{0}^{2}\right)_{\xi}, \\
w_{1}= & \left.\left.-R\left[\frac{z^{4}}{24}-\frac{z^{3}}{6}+\frac{z^{2}}{4}\right] g_{\xi \xi}-G \sigma\right] \frac{z^{3}}{6}-\frac{z^{2}}{2}\right] \eta_{1 \xi \xi} \\
& -\frac{1}{2}\left[\left(R-\frac{16 G \sigma}{3}\right)\left(\frac{z^{3}}{6}+\frac{z^{2}}{2}\right]-G \sigma \frac{z^{2}}{2}\right]\left(\eta_{0}^{2}\right)_{\xi \xi} .
\end{aligned}
$$

In the expressions, $g(\xi, \tau)$ and $\eta_{1}(\xi, \tau)$ are arbitrary functions, which must satisfy

$$
-\frac{R}{8} g_{\xi \xi}+\frac{G \sigma}{3} \eta_{1 \xi \xi}=\eta_{0 \tau}+\left(\frac{R}{6}-\frac{11 G \sigma}{18}\right)\left(\eta_{0}^{2}\right)_{\xi \xi} .
$$

We are essentially interested in determining the evolu- 
tion equation for $\eta_{0}$. To do so, we do not need to calculate $u_{2}, w_{2}, u_{3}, w_{3}, p_{2}$, etc. We need only to obtain $\Theta_{2 z}$ and $\Theta_{3 z}$. Each one of them must satisfy two boundary conditions, Eqs. (9) and (11), implying two compatibility conditions, one at each order. This leads to

$$
\Theta_{2 z}=-\frac{8 G \sigma}{R} z \eta_{0 \xi \xi}+G \sigma\left(\frac{z^{5}}{45}-\frac{5 z^{4}}{72}+\frac{z^{3}}{18}\right) \eta_{0 \xi \xi},
$$

with the compatibility condition

$$
R=320
$$

and

$$
\begin{aligned}
\Theta_{3 z}= & \frac{G \sigma}{120} z \eta_{0 \tau}+\frac{G^{2} \sigma^{2}}{360}\left[\frac{z^{4}}{3}-\frac{5 z^{3}}{6}+\frac{z^{2}}{2}\right]\left(\eta_{0 \xi}\right)^{2} \\
& +\left[\left(80-\frac{4 G \sigma}{3}\right]\left[\frac{z^{4}}{12}-\frac{z^{3}}{3}\right]-\frac{G \sigma}{12} z^{3}\right]\left(\eta_{0}^{2}\right)_{\xi \xi} \\
& +80\left[\frac{z^{5}}{30}-\frac{z^{4}}{6}+\frac{z^{3}}{3}-\frac{z}{80}\right] g_{\xi \xi} \\
& +G \sigma\left[\frac{z^{4}}{24}-\frac{z^{3}}{6}\right]\left(\eta_{1}\right)_{\xi \xi} .
\end{aligned}
$$

Now, using Eqs. (9) and (11), the other compatibility condition emerges, involving $\eta_{1}$ and $g$ in exactly the same combination they appear in Eq. (12). This fact allows the elimination of $\eta_{1}$ and $g$ from these equations, leading to the following evolution equation for $\eta_{0}$ :

$$
\left[\frac{G \sigma}{45}-1\right] \eta_{0 \tau}+\frac{4 G \sigma}{45} \eta_{0} \eta_{0 \xi \xi}+\left[\frac{4 G \sigma}{45}+\frac{4}{3}\right]\left(\eta_{0 \xi}\right)^{2}=0 \text {. }
$$

This is just the evolution equation for $\eta_{0}$ we were looking for, and which appears when $R=320$. Note that this is the critical Rayleigh number for the onset of convective motion, which was found previously in Ref. [1]. Therefore, Eq. (14) describes the time evolution of surface displacements at the critical point for the onset of convection. For each solution $\eta_{0}$ of Eq. (14), the whole lowest order follows, as $u_{0}, w_{0}, p_{0}$, and $\Theta_{0}$ are all functions of $\eta_{0}$

A further simplification comes in if we remember that the validity of the Boussinesq approximation implies $G \sigma \gg 1$, which is indeed the most usual case. Dividing Eq. (14) by $G \sigma$, and neglecting $(G \sigma)^{-1}$ terms in comparison to 1 , it results in

$$
\eta_{0 \tau}+4 \eta_{0} n_{\jmath \xi}+4\left(\eta_{0 \xi}\right)^{2}=0
$$

or in a com pact form

$$
\eta_{0 \tau}+2\left(\eta_{0}^{2}\right)_{\xi \xi}=0 \text {. }
$$

Introducing a new field defined by $\phi=-\eta_{0}$, Eq. (15) becomes

$$
\phi_{\tau}=2\left(\phi^{2}\right)_{\xi \xi},
$$

which is a nonlinear diffusion equation [11].
The self-similar solution to Eq. (16) has been discussed in the literature $[11,14]$, and it can be written in the form

$$
\phi(\xi, \tau)=\left\{\begin{array}{l}
\frac{1}{6}\left[\frac{Q^{2}}{2 \tau}\right]^{1 / 3}\left(\chi_{0}^{2}-\chi^{2}\right) \text { if } \chi<\chi_{0} \\
0 \text { if } \chi>\chi_{0}
\end{array}\right.
$$

where $\chi$ is the self-similar variable defined by

$$
\chi=\frac{\xi}{(2 Q \tau)^{1 / 3}}, \quad Q=\int_{-\infty}^{+\infty} \phi(\xi, 0) d \xi
$$

with $\chi_{0}=\left(\frac{9}{2}\right)^{1 / 3}$. We note that both the evolution equation and its self-similar solution are invariant under the transformation

$$
\xi=\epsilon x, \quad \tau=\epsilon^{3} t, \quad \phi=-\eta_{0}=-\epsilon^{-1} \eta .
$$

Therefore, in stretched as well as in real coordinates, the same solution is valid. Now, solution (17) is highly singular at $\tau=0$, but its interest comes from the fact that it describes the asymptotic behavior for $\tau \rightarrow \infty$. In other words, giving any initial condition for Eq. (16), say $\phi(\xi, 0)$ which is supposed to be a localized function that satisfies $\phi(\xi, 0) \geq 0$, then $\phi(\xi, \tau)$ will tend asymptotically to the solution (17) as $\tau \rightarrow \infty$ [15]. Therefore, Eq. (17) describes the general behavior of the solutions to Eq. (16) for surface perturbation $\eta_{0}$ negative, and for large times. Their most prominent property is the existence of sharp propagating fronts: an initial localized disturbance will always be nonzero over a growing bounded region of the $\xi$ axis. The points where it falls to zero are called fronts. They propagate with opposite finite velocities, corresponding to a noninstantaneous spread out of the disturbance, which is opposite to the typical behavior of processes governed by linear diffusion equations, which would be the case for $R$ well below 320. For $R-320$ sufficiently small, we expect the nonlinear diffusion process to be dominant, leading to the appearance of sharp propagating fronts.

For the sake of completeness, let us note that the $(2+1)$-dimensional version of Eq. (14) can be obtained through the introduction of an $x$-transverse $y$ coordinate from the beginning, and by defining its stretched counterpart according to $\zeta=\epsilon y$. This definition puts the coordinates $x$ and $y$ on the same footing, and the resulting evolution equation, in this case, will be

$$
\begin{aligned}
& {\left[\frac{G \sigma}{45}-1\right] \eta_{0 \tau}+\frac{4 G \sigma}{45} \eta_{0}\left(\eta_{0 \xi \xi}+\eta_{0 \xi \xi}\right)} \\
& +\left[\frac{4 G \sigma}{45}+\frac{4}{3}\right]\left(\eta_{0 \xi}^{2}+\eta_{0 \xi}^{2}\right)=0 .
\end{aligned}
$$

For $G \sigma \gg 1$, it results in

$$
\eta_{0 \tau}+2\left(\eta_{0}^{2}\right)_{\xi \xi}+2\left(\eta_{0}^{2}\right)_{\xi \xi}=0 \text {. }
$$

This equation has also been studied in the literature [14], and in the case of circular symmetry it presents the same kind of sharp propagating fronts as Eq. (15).

We now examine the meaning of negative perturbations. First, we observe that since we are interested in 
studying the evolution of surface or temperature perturbations of the system, it is quite convenient to introduce another Rayleigh number, defined by

$$
\bar{R}=\frac{g \rho_{0} h^{3} \alpha}{\kappa \mu} \Delta T,
$$

where $\Delta T$ is the temperature difference between the bottom and the moving upper surface. Of course, as a consequence of the deformable upper surface, $\bar{R}$ is a nonstationary $x$-dependent Rayleigh number. Using that $\Delta T=(F h / k)+\epsilon(F / k) \eta_{0}+o\left(\epsilon^{2}\right)$, we obtain for $R=320$,

$$
\bar{R}=320+\epsilon \frac{g \rho_{0} h^{3} \alpha F}{\kappa \mu k} \eta_{0}+o\left(\epsilon^{2}\right) .
$$

This new Rayleigh number states the balance between buoyancy and dissipation for each coordinate $x$ and time $t$. Therefore, we can see that a negative $\eta_{0}$ will stand for a perturbation leading to $\bar{R}$ lower than 320 at any $x$ and $t$. In terms of the temperature $\Theta_{0}$, the same is valid for a cooling perturbation. Consequently, for this kind of perturbation, buoyancy is not enough to start the convective motion, and the surface behavior will be described by Eq.
(17). For a positive or a heating perturbation, $\bar{R}$ would be above 320 and the solutions of Eq. (15) would grow indefinitely, showing the inadequacy of the approach for this case. In fact, for positive perturbations the system enters the convective regime, for which the scaling we are using is not appropriate, and the equations we obtained are not valid.

To summarize, we have derived the evolution equation governing negative surface, or cooling perturbations of a shallow fluid, at the critical point for the onset of convection, in a system of the Rayleigh-Bénard type. We have considered specific boundary conditions giving rise to motions with a very large characteristic wavelength. The equation found is a nonlinear diffusion type equation, and in certain instances it may develop solutions with sharp propagating fronts.

The authors would like to thank Conselho Nacional de Desenvolvimento Científico e Tecnológico (CNPq), Brazil, for financial support. One of the authors (M.A.M.) would like to also thank the Instituto de Física Teórica, UNESP, for the kind hospitality, and CNPq for a travel grant.
[1] D. A. Nield, J. Fluid Mech. 29, 545 (1964).

[2] G. B. Whitham, Linear and Nonlinear Waves (Wiley, New York, 1974).

[3] C. J. Chapman and M. R. E. Proctor, J. Fluid Mech. 101, 759 (1980).

[4] T. Taniuti, Suppl. Prog. Theor. Phys. 55, 1 (1974).

[5] G. L. Lamb, Jr., Elements of Soliton Theory (Wiley, New York, 1980).

[6] R. K. Dodd et al., Solitons and Nonlinear Wave Equations (Academic, London, 1982).

[7] E. Infeld and G. Rowlands, Nonlinear Waves, Solitons and Chaos (Cambridge University, Cambridge, 1990).

[8] C. M. Alfaro and M. C. Depassier, Phys. Rev. Lett. 62, 2597 (1989).

[9] R. A. Kraenkel, J. G. Pereira, and M. A. Manna, Phys. Rev. A 45, 838 (1992); S. M. Kurcbart, M. A. Manna, J.
G. Pereira, and A. N. Garazo, Phys. Lett. A 148, 53 (1990).

[10] R. A. Kraenkel, J. G. Pereira, and M. A. Manna, Phys. Rev. A 46, 4786 (1992).

[11] Ya. B. Zel'dovich and Yu. P. Raizer, Physics of Shock Waves and High-Temperature Hydrodynamic Phenomena (Academic, New York, 1967), Vol. II.

[12] J. V. Wehausen and E. V. Laitone, in Handbuch der Physik, edited by S. Flügge (Springer, Berlin, 1960), Vol. 9.

[13] V. L. Gertsberg and G. I. Sivashinsky, Prog. Theor. Phys. 66, 1219 (1981).

[14] W. F. Ames, Nonlinear Partial Differential Equations in Engineering (Academic, New York, 1967).

[15] Ya. B. Zel'dovich and G. I. Barenblatt, Dokl. Akad. Nauk SSSR 41, 671 (1958) [Phys. Dokl. 3, 44 (1958)]. 